\title{
28 Research Square \\ Predictive factors of bowel resection due to an incarcerated groin hernia
}

\author{
Tetsunobu Udaka \\ Mitoyo General Hospital \\ Atsuki Taniguchi \\ Mitoyo General Hospital \\ Jun Kozai \\ Mitoyo General Hospital \\ Tomoaki Ootsuka ( $\nabla$ tom.ohtsuka@mitoyo-hosp.jp ) \\ Nobuyuki Watanabe \\ Mitoyo General Hospital \\ Izuru Endou \\ Mitoyo General Hospital \\ Osamu Yoshida \\ Mitoyo General Hospital \\ Hiroaki Asano \\ Mitoyo General Hospital \\ Masatoshi Kubo \\ Mitoyo General Hospital
}

\section{Research article}

Keywords: groin hernia, prognostic factor, intestinal resection, incarceration, CT attenuation, WBC count

Posted Date: June 18th, 2020

DOI: https://doi.org/10.21203/rs.3.rs-23453/v1

License: (9) This work is licensed under a Creative Commons Attribution 4.0 International License. Read Full License 


\section{Abstract \\ Purpose}

In this retrospective study, we assessed factors that can be used to predict the need for bowel resection due to an incarcerated groin hernia.

\section{Methods}

We enrolled a total of 83 patients diagnosed with an incarcerated groin hernia on preoperative unenhanced computed tomography (CT) between January 2010 and December 2019 in our hospital. They were divided into two groups: those who underwent bowel resection and those who did not. The clinical findings, blood test results, and unenhanced CT values of patients were examined and compared between the two groups. The indication of intestinal resection was macroscopic necrosis or perforation of the incarcerated intestine.

\section{Results}

A total of 83 patients with incarcerated groin hernias were included in our study, of whom $13(15.7 \%)$ had undergone bowel resection surgery. A univariate analysis identified a prolonged time from the symptom onset to surgery, increased white blood cell (WBC) count, increased C-reactive protein (CRP) level, decreased albumin level, and CT attenuation of the incarcerated intestinal wall at the fundus as significant predictive factors. The cut-off value for prediction of intestinal resection was $25 \mathrm{HU}$ for the average CT attenuation of the incarcerated intestinal wall at the fundus and a WBC of 11,550 based on the receiver operating characteristic (ROC) curve. A multivariate analysis showed CT attenuation of the incarcerated intestinal wall at the fundus and an increased WBC count to be independent predictive factors.

\section{Conclusions}

Measuring unenhanced CT attenuation of the incarcerated intestinal wall at the fundus and the WBC count was suggested to be necessary for determining whether or not resection of the incarcerated intestine is required in cases of groin hernia.

\section{Introduction}

Groin hernia, which is a combination of inguinal and femoral hernia, occurs in $27 \%$ of men and $3 \%$ of women [1] and is commonly encountered on a daily basis. Around $5 \%-15 \%$ of hernia patients have incarceration, and approximately $15 \%$ of cases may develop bowel necrosis requiring bowel resection [2]. Patients with bowel necrosis have a significantly longer hospitalization period and a higher rate of 
postoperative complications than those without necrosis, ranging from $6-43 \%$ [3-6], with a mortality rate of $1 \%-7 \%[3,5]$.

If the ischemia in the intestinal tract is reversible, it is common to attempt manual reduction of the incarcerated groin hernia, and if manual reduction of the incarcerated groin hernia is successful, the elective surgery can be performed. However, when intestinal ischemia is irreversible, manual reduction is a risky procedure and emergency surgery is required.

Early recognition of bowel resection risk and timely surgery are critical for ensuring the best outcome of incarcerated groin hernia. Various risk factors for bowel resection have been reported including a female sex, older age, severe coexisting disease, delayed hospitalization, femoral hernia, high American Society Anesthesiologists (ASA) class, presence of peritonitis, and lack of health insurance $[3,5,7,8,9]$. However, some of these factors are interrelated and some cannot be obtained through a regression analysis, which limits the clinical applicability of these results [3]. Furthermore, these factors do not include the unenhanced computed tomography (CT) attenuation.

In the present study, we aimed to investigate the factors that determine the need for intestinal resection before surgery including unenhanced CT attenuation.

\section{Methods}

\section{Patients and Study Design}

The study protocol was approved by the Institutional Review Board of Mitoyo General Hospital, and it conformed to the concepts of the Declaration of Helsinki and its amendments. We enrolled a total of 83 patients who were diagnosed with groin hernia and underwent emergency surgery from January 2010 to December 2019 in our hospital. Patients underwent unenhanced CT and had a measurable CT value of the incarcerated intestinal wall at the fundus.

Patients were divided into two groups according to the operative findings; the bowel resection group included patients found to have an incarcerated small intestine requiring resection, and the non-resection group included patients who did not require small intestine resection. Where or not to perform intestinal resection was determined on intraoperative findings, and intestinal resection was indicated only when clear intestinal necrosis or perforation was found.

The preoperative data gathered were the age, gender, classification of hernia, time from the symptom onset to the surgery, preoperative body temperature, preoperative white blood cell (WBC) count, preoperative C-reactive protein (CRP), preoperative albumin (Alb), preoperative lactate dehydrogenase $(\mathrm{LDH})$, preoperative creatine phosphokinase (CPK), and unenhanced CT value.

For the specific measurement of CT values, CT attenuation of the incarcerated small intestine at the fundus was determined by unenhanced CT at three locations (when possible, the mean values were 
extracted from the horizontal, coronal, and sagittal cross-sectional images) (Fig. 1).

\section{Outcome measures}

The main outcome examined was the factors predictive of an incarcerated small bowel necessitating surgical resection in patients with groin hernia.

\section{Statistical analyses}

Statistical analyses were performed using the Statistical Package for Social Science (SPSS) version 24 (Chicago, IL, USA) software program. The chi-square and Fisher's exact tests were used to compare categorical variables, and Student's $t$-test or the Mann-Whitney test was used to compare continuous variables. Subsequently, receiver operating-characteristic (ROC) curves [9] were constructed to illustrate the sensitivity and false positive rate (1-specificity) of parameters for the prediction of bowel resection. A multiple logistic regression analysis was performed to identify significant predictors associated with the need for bowel resection. All tests were two-sided and $P$ values lower than 0.05 were considered significant.

\section{Results}

\section{A univariate analysis of the risk factors based on resection of the intestine}

A total of 83 patients with incarcerated groin hernias were included in our study, of whom $13(15.7 \%)$ had undergone bowel resection surgery. The mean age was $77.2 \pm 8.6$ years old in the resection group and $76.4 \pm 13.9$ years old in the non-resection group, which was not significantly different. There were also no significant differences in the gender, classification of hernia, or body temperature between the two groups.

The mean time from the symptom onset to the surgery was significantly longer in the resection group $(39.5 \pm 27.8 \mathrm{~h})$ than in the non-resection group $(16.8 \pm 17.7 \mathrm{~h})(\mathrm{P}=0.0014)$. The WBC count and CRP, LDH, and CPK levels were significantly higher in the resection group than in the non- resection group, and Alb was significantly lower in the resection group than in the non-resection group. The average CT attenuation of the incarcerated small intestinal wall at the fundus was $20.65 \pm 3.16 \mathrm{HU}$ in the resection group and $28.12 \pm 3.51 \mathrm{HU}$ in the non-resection group showing a significant difference $(P<0.001)$.

\section{A multivariate analysis of risk factors based on resection of the intestine}

Regarding the CT value, the cut-off value for predicting intestinal resection was $25 \mathrm{HU}$ (sensitivity: 0.923 , specificity: 0.229 , according to the examination of the ROC curve. Figure 2). The results showed that 12 
of 13 patients in the resection group had a CT value of $\leqq 25 \mathrm{HU}$, and 57 of 70 patients in the nonresection group had a CT value of $>25 \mathrm{HU}$.

Regarding the WBC count, the cut-off value for predicting intestinal resection was 11,550 (sensitivity: 0.769 , specificity: 0.200 , according to the examination of the ROC curve. Figure 3 ). The results showed that 10 of 13 patients in the resection group had a WBC count of $\geqq 11,550$, and 56 of 70 patients in the non-resection group had a WBC count of $<11,550$. Similarly, the cut-off values for the time from the symptom onset to surgery, and the CRP, and Alb levels were determined based on the ROC curve (the time from symptom onset to surgery $=22 \mathrm{~h}$, sensitivity: 0.769 , specificity: $0.286, C R P=1.99$, sensitivity: 0.615 , specificity: 0.286 , Alb $=3.95$, sensitivity: 0.769 , specificity: 0.486 ).

A multivariate analysis was performed in the groups classified by CT attenuation of incarcerated intestinal wall at the fundus, WBC count, the time from symptom onset to surgery, and CRP and Alb levels, parameters that were all significantly different in the univariate analysis. The multivariate analysis showed that the odds ratio for the presence of intestinal resection was 32.502 (95\% confidential interval $(\mathrm{Cl})$ : 3.116-339.028) $(P=0.004)$ for the $\mathrm{CT}$ attenuation of the incarcerated intestinal wall at the fundus and $10.153(95 \% \mathrm{Cl}: 1.585-65.045)(P=0.014)$ for the WBC count, which were independent predictors of intestinal resection.

\section{Discussion}

Alvarez et al. [2] observed that 13\% of incarcerated groin hernia patients had undergone bowel resection surgery. Kurt et al. [5] reported that bowel resection was required in approximately $15 \%$ of patents with an incarcerated groin hernia. Suppiah et al. [11] revealed that emergency femoral hernia surgery had a higher rate of bowel resection than did elective femoral hernia surgery. Kemler et al. [12] reported that small bowel resection was required in up to $60 \%$ of patients with strangulated femoral hernias. Therefore, the rate of bowel resection is high in incarcerated groin hernia. In our study of 83 patients with incarcerated groin hernias 13 patents $(15.7 \%)$ underwent bowel resection surgery.

It is very important to understand the preoperative condition of the incarcerated intestinal tract, information that is indispensable for deciding on the surgical technique, such as whether or not to resect the intestinal tract and whether or not to use mesh.

Chen et al. [13] reported in a meta-analysis that the total rate of resection was $21.0 \%$ (160/762) in the patients with incarcerated groin hernia. Eight factors were significantly related to the risk of bowel resection in their pooled analysis: female sex, femoral hernia, bowel obstruction duration of incarceration (hours), WBC count and neutrophilic leukocyte count. Our study indicated that CT attenuation of the incarcerated intestinal wall and the WBC count were significantly associated with the risk of bowel resection in incarcerated groin hernia patients.

In our study, we measured the CT value of the incarcerated intestinal wall at the fundus with the groin hernia patients and examined the need for intestinal resection retrospectively. The CT value is the image 
density value in a two-dimensional CT image. In recent years, there have been some reports on the utility of CT values in the field of abdominal emergency $[14,15]$. Geffroy et al. [15] showed that increased unenhanced bowel-wall attenuation on 64-section multidirector CT had a useful sensitivity and high specificity for the diagnosis of bowel wall ischemia in a highly selected population of patients with surgically treated small bowel obstruction (SBO). Chuong et al. [14] showed that adding unenhanced images to contrast-enhanced images when performing CT to detect bowel wall ischemia based on the presence of decreased bowel wall enhancement in patents with SBO improved the sensitivity, level of confidence in assessing decreased bowl enhancement, and interobserver agreement. In our study, we measured the CT values of the incarcerated small intestine at the fundus by unenhanced CT at three locations (when possible, the mean values were extracted from the horizontal, coronal, and sagittal crosssectional images). The average CT attenuation of the incarcerated small intestinal wall at the fundus was $20.65 \pm 3.16 \mathrm{HU}$ in the resection group and $28.12 \pm 3.51 \mathrm{HU}$ in the non-resection group. Significant differences were observed between two groups. The cut-off value for predicting intestinal resection was $25 \mathrm{HU}$ (sensitivity: 0.923 , specificity: 0.229 , according to the examination of the ROC curve).

Inflammation indicators such as the WBC count, percentage of neutrophils, and neutrophil-to-lymphocyte ratio (NLR) were increased when incarcerated hernia evolved into a strangulated hernia [16-18]. These elevated inflammatory markers suggest a high risk of bowel necrosis, so emergency surgery should be performed immediately. In our study, regarding the WBC count, the cut-off count foe predicting intestinal resection was 11,550 , (sensitivity: 0.769 , specificity: 0.200 , according to the examination of the ROC curve). A multivariate analysis showed that the odds ratio for the presence of intestinal resection was 10.153 (95\% Cl: 1.585 to 65.045$)$ for the WBC count, making it an independent predictor of intestinal resection.

Our study indicated that CT attenuation of incarcerated intestinal wall and the WBC count were significantly associated with the risk of bowel resection in incarcerated groin hernia patients. Several limitations associated with the present study warrant mention. First, this was a retrospective study and our data are based on the medical examinations performed at our hospital. Selection bias therefore could not be completely avoided. Second, due to the single-center setting, this model requires further validation. Further large-scale and well-designed studies are needed.

\section{Conclusions}

In the present study, CT attenuation of the incarcerated intestinal wall at the fundus and the WBC count were useful as predictive factors for intestinal resection of incarcerated groin hernia. Predicting whether or not to resect the intestinal tract before surgery is useful clinically, including for determining the need for resection, the use of mesh, the method of reaching the surgical field, and the need for manual reduction before surgery.

\section{Abbreviations}


ALB

Albumin

$\mathrm{Cl}$

confidential interval

CPK

creatine phosphokinase

CRP

C-reactive protein

CT

Computed tomography

$\mathrm{LDH}$

lactate dehydrogenas

MLK

neutrophil-to-lymphocyte ratio

ROC

Receiver operating-characteristic

SBO

Small bowel obstruction

SIRS

Systemic inflammatory response syndrome

SPSS

Statistical Package for Social Science

WBC

white blood cell

\section{Declarations}

\section{Ethical Approval and Consent to participate}

Not applicable

Consent for publication

Not applicable

\section{Availability of supporting data}

Please contact the authors for data requests

\section{Competing interests}

The authors declare that they have no competing interests. 


\section{Funding}

No funding.

\section{Authors' contributions}

T Udaka designed the study, acquired the data, analyzed and interpreted the data, and drafted and revised the manuscript. $\mathrm{H}$ Asano helped to acquire the data and revised the manuscript. IE, OY, HA, and MK helped to revise the manuscript. All authors read and approved the final manuscript.

\section{Acknowledgements}

Not applicable

\section{References}

1. Primatesta P, Goldacre MJ. Inguinal hernia repair: incidence of elective and emergency surgery, readmission and mortality. Int J Epidemiol. 1996;25:835-9.

2. Dai W, Chen Z, Zuo J, Tan J, Tan M, Yuan Y. Risk factors of postoperative complications after emergency repair of incarcerated groin hernia for adult patients: a retrospective cohort study. Hernia. 2019;23:267-76.

3. Ge BJ, Huang Q, Liu LM, Bian HP, Fan YZ. Risk factors for bowel resection and outcome in patients with incarcerated groin hernias. Hernia. 2010;14:256-64.

4. Koizumi N, Sata N, Kanneda Y, Endo K, Sasanuma H, Sakuma Y, et al. Optimal timeline for emergency surgery in patients with srrangulated groin hernias. Hernia. 2014;18:845-8.

5. Kurt N, Oncel M, Ozkan Z, Bingul S. Risk and outcome of bowel resection in patients with incarcerated groin hernas: retrospective study. Word J Surg. 2003;27:741-3.

6. Tanaka N, Uchida N, Ogihara H, Sasamoto H, Kato H, Kuwano H. Clinical study of inguinal and femoral incarcerated hernias. Surg Today. 2010;40:1144-7.

7. Mizrahi H, Parker MC. Management of asymptomatic inguinal hernia: a systemic review of the evidence. Arch Surg. 2012;147:277-81.

8. Alvarez JA, Baldonedo RF, Bear IG, Solis JA, Alvarez P, Jorge JL. Incarcerated groin hernias in adults: presentation and outcome. Hernia. 2004;8:121-6.

9. Abd Ellatif ME, Negm A, Elmorsy G, Al-Katary M, Yousef Ael A, Ellaithy R. Feasibility of mesh repair for strangulated abdominal wall hernias. Int J Surg. 2012;10:153-6.

10. Akobeng AK. Understanding diagnostic tests 3: receiver operating characteristic curves. Acta Paediatr. 2007;96:644-7.

11. Suppiah A, Gatt M, Barandiaran J, Heng MS, Perry EP. Outcomes of emergency and elective femoral hernia surgery in four district general hospitals: a 4-year study. Hernia. 2007;11:509-12. 
12. Kemler MA, Oostvogel HJ. Femoral hernia: is a conservative policy justified? Eur J Surg. 1997;163:187-90.

13. Chen P, Hung L, Yang W, He D, Liu X, Wang Y, et al. Risk factors for bowel resection among patients with incarcerated groin hernias: a meta-analysis. Am J Emerg Med. 2019; Nov 15. pii: S07356757(19)30606-0. doi: 10.1016/j.ajem.2019.09.023. [Epub ahead of print] Review.

14. Chuong AM, Corno L, Beaussier H, Boulay-Coletta I, Millet I, Hodel J, et al. Assessment of bowel wall enhancement for the diagnosis of intestinal ischemia in patients with small bowel obstruction: value of adding unenhanced CT to contrast-enhanced CT. Radiology. 2016;280:98-107.

15. Geffroy Y, Boulay-Coletta I, Jullès MC, Nakache S, Taourel P, Zins M. Increased unenhanced bowelwall attenuation at multidirector $\mathrm{CT}$ is highly specific of ischemia complicating small-bowel obstruction. Radiology. 2014;270:159-67.

16. Zhou H, Ruan X, Shao X, Huang X, Fang G, Zheng X. Clinical value of the neutrophil/lymphocyte ratio in diagnosing adult strangulated inguinal hernia. Int $J$ surgery. 2016;36:76-80.

17. Xie X, Feng S, Tang Z, Chen L, Hung Y, Yang X. Neutrophil-to-lymphocyte ratio predicts the severity of incarcerated groin hernia. Mred Sci Monit. 2017;23:5558-63.

18. Köksal H, Ateş A, Nazik EE, Küçükosmanoğlu I, Doğan SM, Doğru O. Predictive value of preoperative neutrophil-to-lymphocyte ratio while detecting bowel resection in hernia with intestinal incarceration. Ulus Travima Acil Cerrahi Derg. 2018;24:207-10.

\section{Tables}

Due to technical limitations, Tables 1-2 are provided in the Supplementary Files section.

\section{Figures}



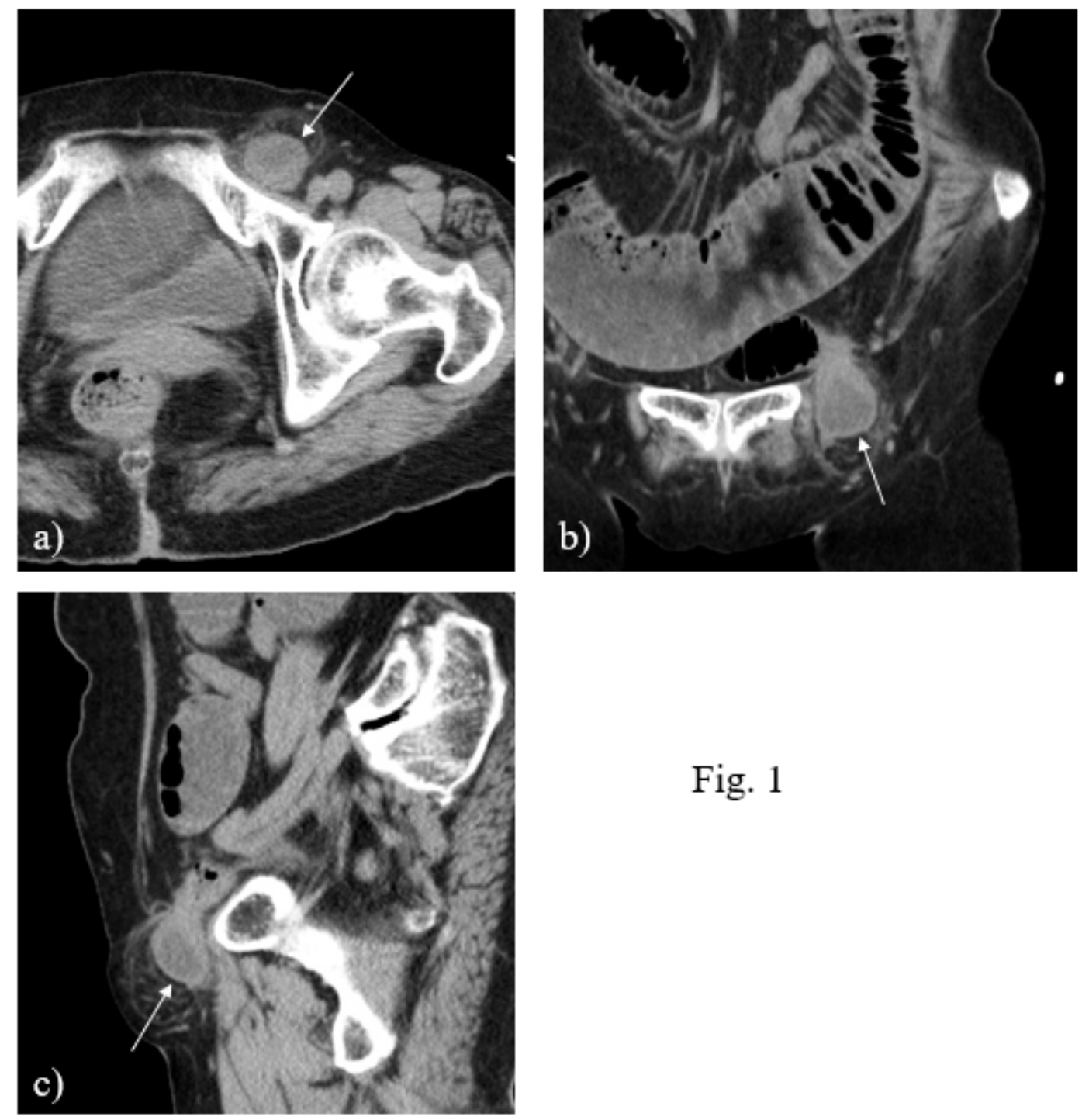

Fig. 1

\section{Figure 1}

Unenhanced CT. (a) Incarcerated intestinal wall of the fundus: horizontal image (Arrow). (b) Incarcerated intestinal wall of the fundus: coronal image (Arrow). (c) Incarcerated intestinal wall of the fundus: sagittal image (Arrow). 


\section{ROC curve}

\section{CT attenuation value of intestinal wall}

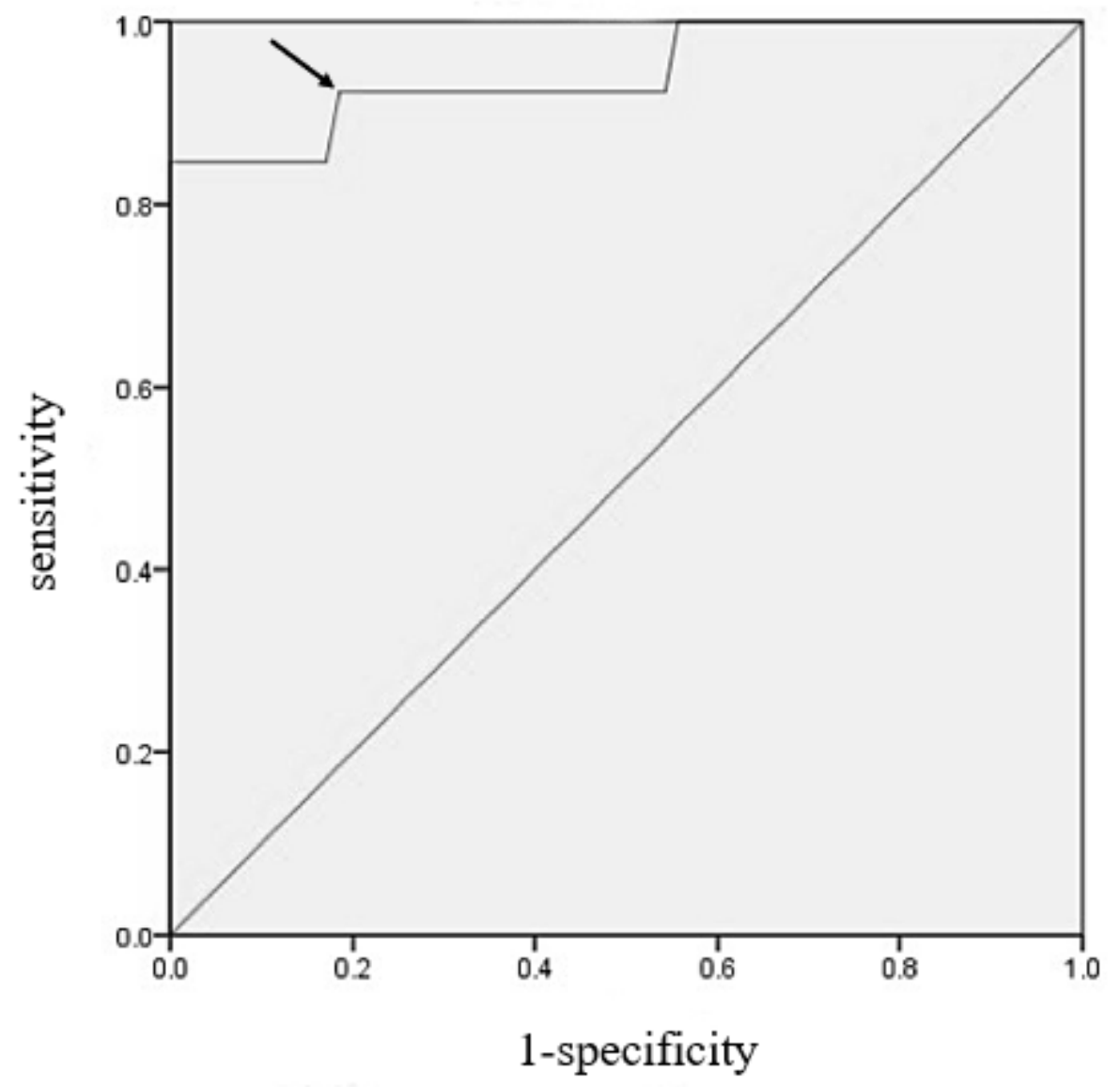

Cut off point $25 \mathrm{HU}$

Sensitivity 0.923

Specificity 0.229

\section{Figure 2}

ROC curve for determining the cut-off CT attenuation value in the presence of an incarcerated intestinal wall of the fundus. 
ROC curve

WBC count

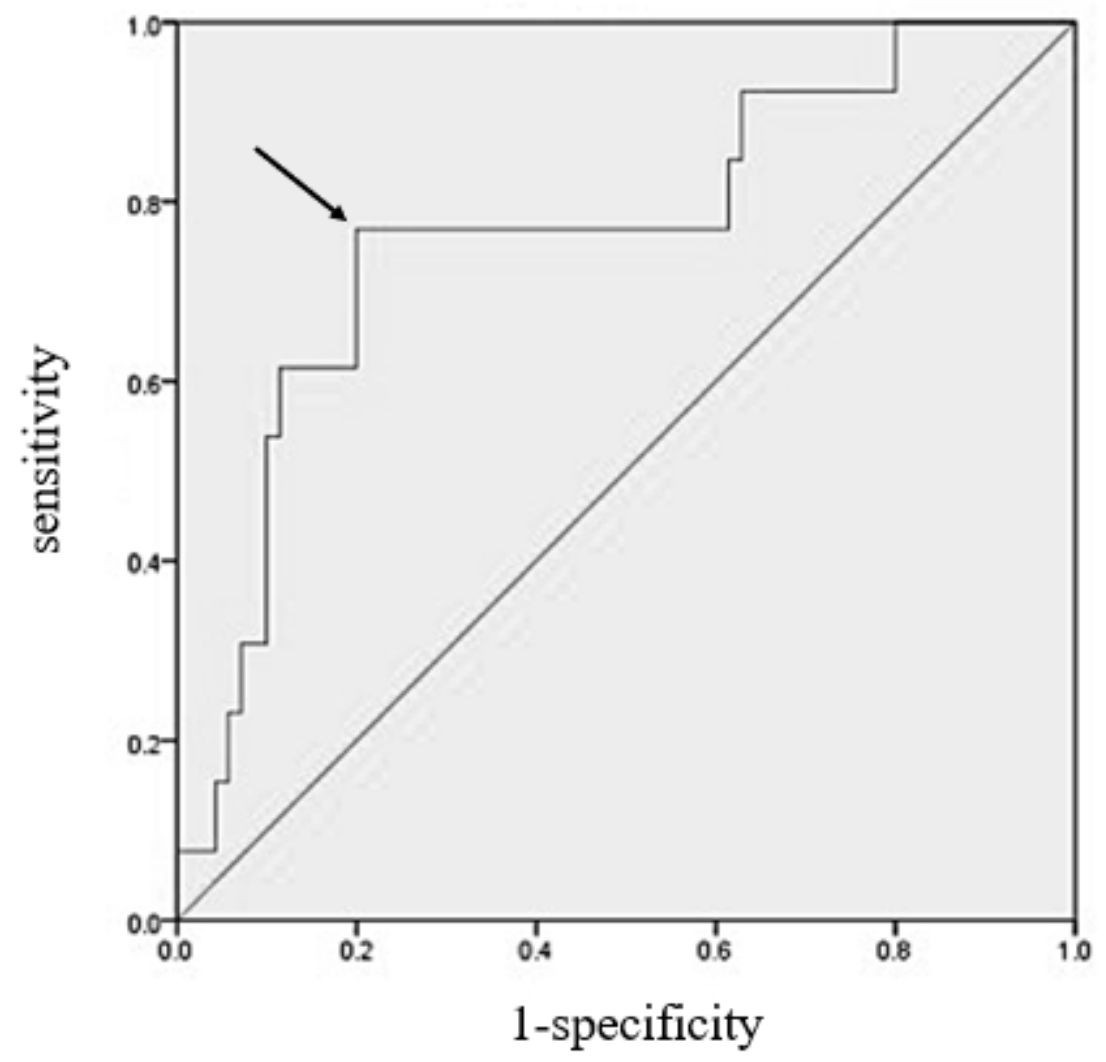

Cut off point 11,550 Sensitivity 0.769 Specificity 0.200

Figure 3

ROC curve for determining the cut-off WBC count.

\section{Supplementary Files}

This is a list of supplementary files associated with this preprint. Click to download.

- Table1.xlsx

- Table2.xlsx 